\title{
An Improved Approach for AC-DC Power Flow Calculation With Multi-Infeed DC Systems
}

\author{
Chongru Liu, Member, IEEE, Boming Zhang, Fellow, IEEE, Yunhe Hou, Member, IEEE, Felix F. Wu, Fellow, IEEE, \\ and Yingshang Liu
}

\begin{abstract}
An improved approach based on sequential method for the AC-DC power flow calculation is proposed in this paper. This approach solves the convergence problem caused by voltage violations at $\mathrm{AC}$ buses during the power flow calculation for the DC subsystems. The convergence property can be significantly improved by adjusting the converter transformer tap position flexibly. In order to adjust the tap position of the converter transformer flexibly, three mainly modifications are proposed. Firstly, the equations for whole DC systems are decoupled into individual DC systems so as to easily figure out which DC system's tap position needs adjustment. Secondly, the tap ratio of a converter transformer is selected as an alternative state variable to replace the cosine of the control angle when necessary. Thirdly, the Newton-Raphson method is utilized to solve DC subsystems instead of the method using the linear equations. Furthermore, a theoretical analysis of the advantages of the proposed approach is also presented. Numerical simulations and practical applications show that the proposed approach meet the requirement of different system operating conditions and has advantages in terms of convergence and speed. The proposed approach has been successfully integrated into the Energy Management System (EMS) for China Southern Power Grid.
\end{abstract}

Index Terms-AC-DC, HVDC transmission, multi-infeed DC systems, Newton-Raphson method, power flow analysis.

\section{INTRODUCTION}

C HINA has made remarkable strides in high voltage direct current (HVDC) transmission implementation. At present, there are more than ten HVDC lines in operation in China. By 2020, the power grid of China will become a robust AC-DC system with ultra high voltage links. China will have 33 HVDC lines by then. North China Grid and Central China Grid will become AC-DC hybrid power systems with multi-infeed DC systems. Furthermore, the complexity of East China Grid,

Manuscript received March 03, 2010; revised July 02, 2010; accepted August 06, 2010. Date of publication September 16, 2010; date of current version April 22, 2011. This work was supported in part by the Special Fund of the National Key Technology R\&D Program of China (2008BAA14B05) and National Natural Science Foundation of China (50807013) and in part by "111" Project (B08013) of China. Paper no. TPWRS-00165-2010.

C. Liu is with the School of Electrical and Electronic Engineering, North China Electric Power University, Beijing, China, and also with the State Key Laboratory of Power System, Tsinghua University, Beijing, China (e-mail: chongru.liu@ncepu.edu.cn).

B. Zhang is with the Electrical Engineering Department, Tsinghua University, Beijing, China (e-mail: zhangbm@ @tsinghua.edu.cn).

Y. Hou and F. F. Wu are with the Electrical Engineering Department, University of Hong Kong, Hong Kong, China (e-mail: yhhou@eee.hku.hk; ffwu@eee. hku.hk).

Y. Liu is with the Department of Production and Technology of China Southern Power Grid Company, Guangzhou, China (e-mail: liuys@csg.cn).

Color versions of one or more of the figures in this paper are available online at http://ieeexplore.iee.org.

Digital Object Identifier 10.1109/TPWRS.2010.2068579

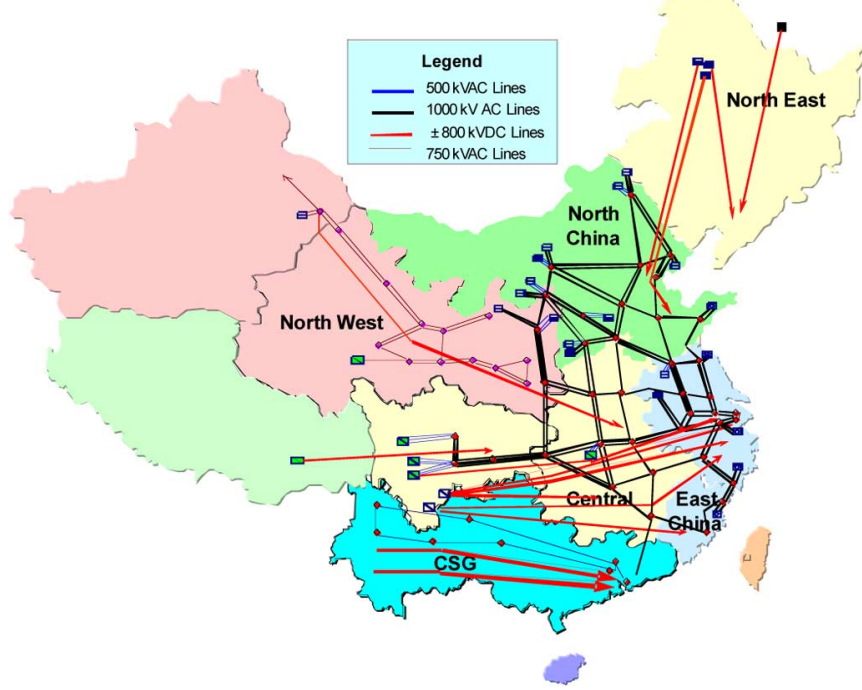

Fig. 1. The layout of the ultra voltage power system of China by 2020 .

China Southern Power Grid (CSG), and Central China Grid will significantly increase as more DC links are connected. The layout of the ultra-high voltage power system in China by 2020 is shown in Fig. 1.

Multi-infeed DC systems have significantly increased the complexity of power flow calculation. Power flow calculation is the fundamental engine in all functions of EMS and requires continuous availability. As a result, the speed and robustness of power flow calculation of complex AC-DC systems are critical to China's power system operation.

Efficient power flow calculation of AC-DC system has drawn much attention lately [1], [2]. The methods can be divided into two categories [3]-[5]: simultaneous method and sequential method. The simultaneous method solves the equations of the complex AC and DC systems simultaneously [6], [7]. However, this approach is incapable to make use of an existing AC power flow program. The sequential method [8] solves AC and DC systems separately. The advantages of this approach are: 1) different algorithms can be applied to the $D C$ and $A C$ systems independently, 2) the software development is easier, 3) the existing AC system power flow program can be used, and (4) it has a lower demand of memory space. Thus, the sequential method is commonly used in the practical EMS for AC-DC power system.

However, when the DC system is operated under the reduced voltage level and/or reduced power condition, the sequential method may encounter the following serious problems:

- When the DC power flow equations are derived directly from the external characteristics of converters and network 
equations, the calculation of reactive power of converters may lead to imaginary numbers [9], [10];

- Iterations may be interrupted because $\cos \theta$ (the cosine value of the firing angle for rectifier or extinction angle for inverter) may become greater than one [11];

- Inappropriate adjustment of converter transformer taps may lead to oscillations between AC and DC systems. The AC-DC load flow calculation may become hard to converge; etc.

To enhance the robustness of the sequential method of AC-DC power flow, an improved method for multi-infeed DC systems is presented in this paper. This method has been implemented in the EMS in CSG and has been running routinely every 5 min since 2007. This improved method has been proved to be fast and robust.

The remaining parts of the paper are organized as follows. Section II describes the models of the proposed method in detail. Section III gives the theoretical justification of this approach. Section IV shows some simulation results. Section V draws the conclusions of this paper.

\section{Models OF THE IMPROVED AC-DC POWER Flows}

\section{A. Advantages of the Improved Approach}

An efficient AC-DC power flow calculation method should overcome the challenges coming from the three serious problems mentioned in Section I. To deal with these problems, an approach based on proper adjustment of the converter transformer tap position is proposed in this paper.

The proposed approach is able to widen the solution space to facilitate iteration convergence in the following manners by modifying traditional algorithms:

- The equations of whole DC systems are decoupled into individual DC systems so as to establish the mathematical model separately. By this means, it is easy to figure out which DC system's tap position needs adjustment.

- To avoid the impractical scenarios caused by $\cos \theta \geq 1$, the $T$ (the tap ratio of converter transformer) is selected as an alternative state variable to replace $\cos \theta$ when necessary.

- The Newton-Raphson (N-R) method is utilized to solve DC subsystems instead of the method using the linear equations derived from external characteristics of converters, control functions, and network equations [8]. With the exchange of the state variables for tap adjustments, the N-R method is more efficient than linear equations.

One advantage of the proposed method is that it can adjust the tap position of converter transformer flexibly and efficiently during power flow calculation, which results in a fast computation. A mathematical proof of the advantages of the method is presented in Section III. Details of this approach are elaborated below.

\section{B. Model of Individual DC System}

In this paper, three state variables and three balance equations are employed for each converter. The direct voltage $V_{d i}$, the direct current $I_{d i}$, and the cosine value of the control angle $\cos \theta_{i}$ (denoted as $A_{i}$ ) are chosen as the state variables for the $i$ th converter, i.e., $x_{\mathrm{dc}}^{i}=\left[V_{d i}, I_{d i}, A_{i}\right]^{\prime}$, where the apostrophe $\left([]^{\prime}\right)$ denotes the transpose of the vector. When $A_{i}$ is chosen as a state variable, the tap ration $T_{i}$ is treated as a known constant during calculation. For example, if the $k$ th DC system unit is a two-terminal DC system composed of the $i$ th converter and the $j$ th converter, the state variables of this DC system are

$$
\boldsymbol{x}_{d c k}=\left[\begin{array}{l}
\boldsymbol{x}_{\mathrm{dc}}^{i} \\
\boldsymbol{x}_{\mathrm{dc}}^{j}
\end{array}\right]=\left[V_{d i}, I_{d i}, A_{i}, V_{d j}, I_{d j}, A_{j}\right]^{\prime} .
$$

For the $i$ th converter, the balance equations are

$$
\left\{\begin{array}{l}
f_{k i 1}\left(x_{d c k}\right)=0 \\
f_{k i 2}\left(x_{d c k}\right)=0 \\
f_{k i 3}\left(x_{d c k}\right)=0
\end{array}\right.
$$

where

$$
\begin{aligned}
& f_{k i 1}\left(x_{d c k}\right) \\
& =V_{d i}-\frac{3 \sqrt{2}}{\pi} B_{i} T_{i} U_{a i} A_{i}+\frac{3}{\pi} X_{c i} I_{d i} \zeta_{i}=0 \\
& f_{k i 2}\left(x_{d c k}\right) \\
& =I_{d i}-\sum_{j \in \Omega_{i}} \frac{V_{d i}-V_{d j}}{R_{d i j}}=0 \\
& \begin{cases}\text { if CP then } & f_{k i 3}\left(x_{d c k}\right)=V_{d i} I_{d i}-P_{d i}^{\text {set }}=0 \\
\text { if CC then } & f_{k i 3}\left(x_{d c k}\right)=I_{d i}-I_{d i}^{\text {set }}=0 \\
\text { if CV then } & f_{k i 3}\left(x_{d c k}\right)=V_{d i}-V_{d i}^{\text {set }}=0 \\
\text { if CA then } & f_{k i 3}\left(x_{d c k}\right)=A_{i}-A_{i}^{\text {set }}=0\end{cases}
\end{aligned}
$$

where $B_{i}$ is the bridge number of the $i$ th converter, $U_{a i}$ is the voltage magnitude on $\mathrm{AC}$ side calculated from $\mathrm{AC}$ power flow, $X_{c i}$ is the equivalent reactance, $\zeta_{i}$ is 1 for rectifier and -1 for inverter. The positive direction of direct current is from converter to DC line, and the positive direction of direct power is from the AC system to the DC system. $\Omega_{i}$ is the set of all converters connected to the $i$ th converter, $R_{d i j}$ is the resistance of the DC line between the $i$ th converter and the $j$ th converter. $f_{k i 3}\left(x_{d c k}\right)$ varies with the control mode of converters. Four commonly used control modes are constant power (CP), constant current (CC), constant voltage (CV), and constant ignition angle for rectifier and constant extinction angle for inverter (CA). The superscript set indicates that the value of the labeled parameter is presetted. That is to say, the parameters with the superscript set are known. In an HVDC system, there are two converters, each of which has an individual operation mode.

For example, if in the $k$ th DC system, the $i$ th converter and the $j$ th converter are operated in CP mode and CA mode, respectively, the calculation equations are

$$
\left\{\begin{array}{l}
f_{k i 1}\left(\boldsymbol{x}_{d c k}\right)=V_{d i}-\frac{3 \sqrt{2}}{\pi} B_{i} T_{i} U_{a i} A_{i}+\frac{3}{\pi} X_{c i} I_{d i} \zeta_{i}=0 \\
f_{k i 2}\left(\boldsymbol{x}_{d c k}\right)=I_{d i}-\frac{V_{d i}-V_{d j}}{R_{d i j}}=0 \\
f_{k i 3}\left(\boldsymbol{x}_{d c k}\right)=V_{d i} I_{d i}-P_{d i}^{s e t}=0 \\
f_{k j 1}\left(\boldsymbol{x}_{d c k}\right)=V_{d j}-\frac{3 \sqrt{2}}{\pi} B_{j} T_{j} U_{a j} A_{j}+\frac{3}{\pi} X_{c j} I_{d j} \zeta_{j}=0 \\
f_{k j 2}\left(\boldsymbol{x}_{d c k}\right)=I_{d j}-\frac{V_{d j}-V_{d i}}{R_{d i j}}=0 \\
f_{k j 3}\left(\boldsymbol{x}_{d c k}\right)=A_{d j}-A_{d j}^{s e t}=0 .
\end{array}\right.
$$


For each DC system, the number of equations is three times of the number of converters. For the $k$ th DC system, the corresponding equations can be written in the following vector form:

$$
\boldsymbol{f}_{k}\left(\boldsymbol{x}_{d c k}\right)=\mathbf{0}
$$

When the operating parameters of a certain system are impractical, they will not affect other DC systems during calculation. Therefore, it is convenient to figure out which DC system does not converge. Furthermore, this decoupled method can easily incorporate new DC lines.

\section{Tap Adjustment Algorithm}

Traditionally, there are two kinds of methods to deal with the tap position of converter transformer: 1) fixing the tap position as a preset value [6], [8]; 2) adjusting the tap position one gear at each iteration [10], [12]. As a result, the calculation might fail to converge because of the existence of an improper tap position, and it is difficult to find out the proper position for that tap.

In this paper, we propose to change the state variable from $A$ to $T$ to deal with the adjustment of tap positions of converter transformers. By this means, it is convenient to find out the proper value of $T$ and facilitate the convergence of the AC-DC load flow calculation. Furthermore, the power flow calculation of AC-DC system becomes robust and can resolve the three problems mentioned in Section I.

The adjustment of tap position is fulfilled after the DC system power flow calculation converges. The tap adjustment algorithm is presented as follows:

- $A_{i}$, i.e., $\cos \theta_{i}$, is checked whether it is less than 1 . If $A_{i}<$ 1 , the calculation of this DC system is over.

- If $A_{i} \geq 1$, the state variables are changed from $\left[V_{d i}, I_{d i}, A_{i}\right]^{\prime}$ to $\left[V_{d i}, I_{d i}, T_{i}\right]^{\prime}$. That means, $T_{i}$ is relaxed as a variable and $A_{i}$ is fixed as a constant when $A_{i} \geq 1$.

- The value of $A_{i}$ is replaced by its value in the last iteration or a specific preset value.

- To obtain the discrete value of $T$, it is regulated to the closest gear as follows:

$$
\Delta T_{i}=\left\lceil\Delta T_{i}^{\prime} / T_{\text {step }}\right\rceil \cdot T_{\text {step }}
$$

where $i$ is the index of the converter, $\Delta T_{i}$ is the actual correction value, \lceil\rceil is the ceiling function, $\Delta T_{i}^{\prime}$ is the calculated value, and $T_{\text {step }}$ is the step length of tap changer of the converter transformer.

This new algorithm avoids the oscillation problem due to irrational adjustments of transformer ratio. It adapts to practical operating scenarios with various modes of HVDC systems, and it has been proved to be fast and accurate in CSG application. Furthermore, even under extreme operation conditions, such as in $\mathrm{N}-1$ contingent cases, the system can be solved by the proposed algorithm.

\section{Newton-Raphson Method for DC System}

The N-R method is more efficient for solving DC equations under changing state variable. Compared with the conventional

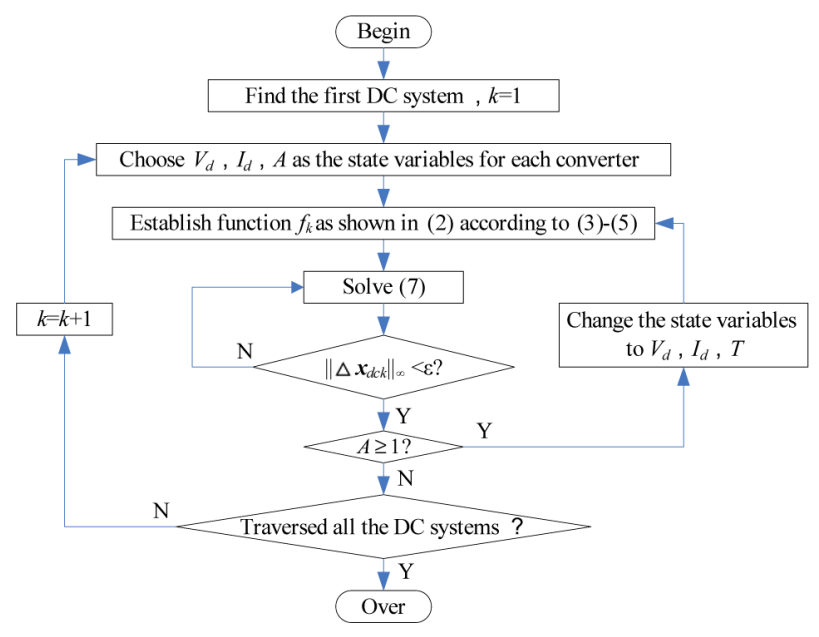

Fig. 2. Flow chart of DC subsystems power flow.

linear methods, it can avoid reconstructing and recalculating the equations from the very beginning when one of the state variables is changed. In addition, the N-R method also enjoys the advantage of superlinear convergence.

By using N-R method to solve (7), the updating formula for the $l$ th iteration is

$$
\left\{\begin{array}{l}
\Delta \boldsymbol{x}_{d c k}^{(l+1)}=\boldsymbol{J}\left(\boldsymbol{x}_{d c k}^{(l)}\right)^{-1} \boldsymbol{f}_{k}\left(\boldsymbol{x}_{d c k}^{(l)}\right) \\
\boldsymbol{x}_{d c k}^{(l+1)}=\boldsymbol{x}_{d c k}^{(l)}-\Delta \boldsymbol{x}_{d c k}^{(l+1)}
\end{array}\right.
$$

where $\boldsymbol{J}\left(\boldsymbol{x}_{d c k}^{(l)}\right)$ is the Jacobian matrix of $\boldsymbol{f}_{k}\left(\boldsymbol{x}_{d c k}^{(l)}\right)$.

Mathematically, if the $i$ th converter is operated in CA control mode, certain row of the $\boldsymbol{J}$ might be zeros after the state variable $A_{i}$ is replaced by $T_{i}$. However, because $A_{i}$ is always less than 1 in CA control mode, the state variable vector is fixed to $\left[V_{d i}, I_{d i}, A_{i}\right]^{T}$. From (2)-(5), it is obvious that at least one of the partial derivatives of $f_{k i 3}\left(x_{d c k}\right)$ with respect to state variables is nonzero.

\section{E. Flowchart of DC Subsystem Calculation}

The flowchart of DC subsystem calculation is shown in Fig. 2.

In most circumstances, the transformer ratio $T$ does not need to be adjusted. However, when the DC system operates under reduced voltage or reduced power, or even when $\mathrm{N}-1$ contingency scan is done over the whole system, adjustment on $T$ is needed to fulfill the power flow calculation.

After the convergence of the DC system power flow, the active power and reactive power of DC system can be calculated as follows:

$$
\left\{\begin{array}{l}
P_{d i}=V_{d i} I_{d i} \\
Q_{d i}=I_{d i} \zeta_{i} \sqrt{V_{d 0 i}^{2}-V_{d i}^{2}}
\end{array}\right.
$$

\section{F. AC Subsystem Power Flow Equations}

The balance equations of the AC buses are as follows:

$$
\boldsymbol{f}\left(\boldsymbol{x}_{\mathrm{ac}}\right)=\left[\begin{array}{l}
\boldsymbol{P}^{\mathrm{spec}}-\boldsymbol{P}^{\mathrm{ac}}\left(\boldsymbol{U}_{a}, \boldsymbol{\theta}_{a}\right)-\boldsymbol{P}^{\mathrm{dc}}\left(\boldsymbol{U}_{a}, \boldsymbol{x}_{\mathrm{dc}}\right) \\
\boldsymbol{Q}^{\mathrm{spec}}-\boldsymbol{Q}^{\mathrm{ac}}\left(\boldsymbol{U}_{a}, \boldsymbol{\theta}_{a}\right)-\boldsymbol{Q}^{\mathrm{dc}}\left(\boldsymbol{U}_{a}, \boldsymbol{x}_{\mathrm{dc}}\right)
\end{array}\right]
$$




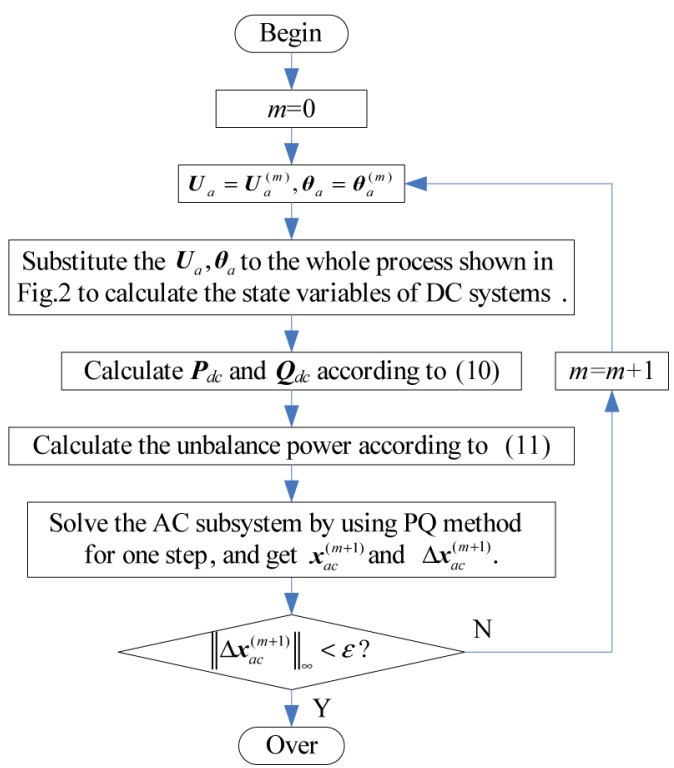

Fig. 3. Sequential method of AC-DC power flow calculation.

where $\boldsymbol{P}^{\text {spec }}$ and $\boldsymbol{Q}^{\text {spec }}$ are the vectors of injected active and reactive power on buses, $\boldsymbol{U}_{a}$ and $\boldsymbol{\theta}_{a}$ are the voltage magnitude and the phase angle, $\boldsymbol{x}_{\mathrm{ac}}$ is the state variable vector of AC systems, $\boldsymbol{P}^{\mathrm{ac}}\left(\boldsymbol{U}_{a}, \boldsymbol{\theta}_{a}\right)$ and $\boldsymbol{Q}^{\mathrm{ac}}\left(\boldsymbol{U}_{a}, \boldsymbol{\theta}_{a}\right)$ are branch power flows of AC systems, $\boldsymbol{x}_{\mathrm{dc}}$ is the state variable vector of DC systems, $\boldsymbol{P}^{d c}\left(\boldsymbol{U}_{a}, \boldsymbol{x}_{\mathrm{dc}}\right)$ and $\boldsymbol{Q}^{\mathrm{dc}}\left(\boldsymbol{U}_{a}, \boldsymbol{x}_{\mathrm{dc}}\right)$ are the active and reactive power transmitted from AC systems to DC systems.

For PQ decoupled method, the calculation may diverge because the traditional power flow calculation for DC system is not robust enough. As a result, in the traditional AC-DC power flow calculation by PQ decoupled method, the constant Jacobian matrices $\boldsymbol{B}^{\prime}$ and $\boldsymbol{B}^{\prime \prime}$ [11] exclusive of DC systems have to be modified to include contribution of DC system on $\boldsymbol{B}^{\prime}$ and $\boldsymbol{B}^{\prime \prime}$. However, when the proposed approach is applied, the power flow calculations become robust and the convergence is improved. In order to keep the constant structure of the Jacobian matrices $\boldsymbol{B}^{\prime}$ and $\boldsymbol{B}^{\prime \prime}$, only the balance equations, instead of both Jacobian matrices and balance equations, are modified during the AC-DC power flow calculation.

The core idea of the sequential method for AC-DC system power flow calculation is to add a calculation procedure for the DC system into each iteration of the AC system calculation, as shown in Fig. 3, where $m$ is the iteration times for the AC system power flow calculation.

\section{THEORETICAL ANALYSIS}

\section{A. Advantages of the Revised Algorithm}

The advantages of the approach presented in this paper are as follows:

1) Ensure $A_{i}<1: A_{i}$ is the cosine value of the control angle of the $i$ th converter, which must be less than 1 . Obviously, $A_{i}<1$ is ensured in the process by fixing it to its value in the previous iteration or a preset value whenever $A_{i} \geq 1$.

2) Ensure $V_{d 0 i}>V_{d i}$ : For the $i$ th converter, the following equality holds:

$$
V_{d i}=V_{d 0 i} A_{i}-\frac{3}{\pi} X_{c i} I_{d i} \zeta_{i}
$$

where

$$
V_{d 0 i}=\frac{3 \sqrt{2}}{\pi} B_{i} T_{i} U_{a i}
$$

Equation (12) can be rewritten as

$$
A_{i}=\frac{V_{d i}+\frac{3}{\pi} X_{c i} I_{d i} \zeta_{i}}{V_{d 0 i}} .
$$

Because $A_{i}<1$ and $I_{d i} \zeta_{i}=\left|I_{d i}\right|$, the following inequality holds:

$$
V_{d 0 i}>V_{d i}+\frac{3}{\pi} X_{c i} I_{d i} \zeta_{i}>V_{d i} .
$$

For the $i$ th converter, the ideal no-load voltage $V_{d 0 i}$ is ensured to be higher than the direct voltage $V_{d i}$. Hence from (10), we have avoided calculating the square root of a negative number by ensuring $V_{d 0 i}>V_{d i}$.

3) Widen the Solution Space to Facilitate Iteration Convergence: From (3)-(5), $T_{i} A_{i}$ can be directly expressed as follows:

$$
T_{i} A_{i}=\frac{\pi V_{d i}}{3 \sqrt{2} B_{i} U_{a i}}+\frac{P_{d i}}{\sqrt{2} B U_{a i} V_{d i}} X_{c i} .
$$

To study the characteristics of $T_{i} A_{i},(16)$ is rewritten as follows with the per unit value $B_{i}=1$ :

$$
g\left(V_{d i}, U_{a i}, P_{d i}, X_{c i}\right) \equiv T_{i} A_{i}=\frac{\pi V_{d i}}{3 \sqrt{2} U_{a i}}+\frac{P_{d i} X_{c i}}{\sqrt{2} U_{a i} V_{d i}} .
$$

The partial derivatives of (17) may be calculated

$$
\begin{aligned}
\frac{\partial g}{\partial V_{d i}} & =\frac{\pi}{3 \sqrt{2} U_{a i}}-\frac{P_{d i} X_{c i}}{\sqrt{2} U_{a i} V_{d i}^{2}} \\
\frac{\partial g}{\partial U_{a i}} & =-\frac{\pi V_{d i}}{3 \sqrt{2} U_{a i}^{2}}-\frac{P_{d i} X_{c i}}{\sqrt{2} V_{d i} U_{a i}^{2}} \\
\frac{\partial g}{\partial P_{d i}} & =\frac{X_{c i}}{\sqrt{2} U_{a i} V_{d i}} \\
\frac{\partial g}{\partial X_{c i}} & =\frac{P_{d i}}{\sqrt{2} U_{a i} V_{d i}} .
\end{aligned}
$$

In practice, when $V_{d i}$ is less than 0.7, the Voltage Dependent Current Order Limiter is applied. The external characteristics function (3) could not be used in this scenario. Hence, for power flow calculation of AC-DC systems, $V_{d i}$ can be assumed larger than 0.7. Furthermore, under steady-state situation, we have $P_{d i}<1$ and $X_{c i} \ll 1$. Consequently, we have

$$
\frac{\partial g}{\partial V_{d i}}>0, \frac{\partial g}{\partial U_{a i}}<0, \frac{\partial g}{\partial P_{d i}}>0, \frac{\partial g}{\partial X_{c i}}>0 .
$$

Equation (17) is a continuous and monotonic function. Therefore, we can get the expression of the upper and lower boundaries of $T_{i} A_{i}$ :

$$
\begin{aligned}
& \min \left(T_{i} A_{i}\right)=g\left(V_{d i}^{\min }, U_{a i}^{\max }, P_{d i}^{\min }, X_{c i}^{\min }\right) \\
& \max \left(T_{i} A_{i}\right)=g\left(V_{d i}^{\max }, U_{a i}^{\min }, P_{d i}^{\max }, X_{c i}^{\max }\right)
\end{aligned}
$$

where

$$
\left\{\begin{array}{l}
V_{d i}^{\min } \leq V_{d i} \leq V_{d i}^{\max } \\
U_{a i}^{\min } \leq U_{a i} \leq U_{a i}^{\max } \\
P_{d i}^{\min } \leq P_{d i} \leq P_{d i}^{\max } \\
X_{c i}^{\min } \leq X_{c i} \leq X_{c i}^{\max } .
\end{array}\right.
$$


Practically, the step length of transformer tap is 0.025 with \pm 8 gears and the ratio can be changed from 0.8 p.u. to 1.2 p.u. In traditional methods, if $\max \left(T_{i} A_{i}\right)>T_{i, \min }$, then $\exists T_{i}^{*} \in$ $\left[T_{i, \min }, T_{i, \max }\right]$ such that $A_{i}=\left(T_{i} A_{i}\right) /\left(T_{i}^{*}\right)>1$. This will lead to unreasonable $A_{i}$. By the proposed method, $A_{i}$ is fixed and $T_{i}$ is loosened.

Empirically, $P_{d i}<1$ and $X_{c i} \ll 1$, and $U_{a i}$ is near 1.0. Therefore, we have the following inequality between $\max \left(T_{i} A_{i}\right)$ and $T_{i, \max }$ :

$$
\max \left(T_{i} A_{i}\right) \leq T_{i, \max }
$$

From (26), $T_{i}$ is always feasible for the solution and can keep $A_{i}$ reasonable. Therefore, the calculation can have great robustness by specifying $T_{i}$ as a state variable instead of $A_{i}$. Consequently, the approach to adjust $T_{i}$ and find out a proper gear is effective and valid in dealing with the situation when $A_{i} \geq 1$. The proposed approach can therefore widen the solution space and improve the convergence of AC-DC power flow calculation.

4) Represent the Characteristics of the DC Control System: There are two types of control in DC system: grid/gate control to the valve ignition angle and the tap changing control to the converter transformer [10]. The grid/gate control, which is rapid (1 to $10 \mathrm{~ms}$ ), and the tap changing, which is slow (5 to 6 s per step), are used in a complementary manner [10]. If the AC bus voltages fluctuate and the grid/gate cannot keep the power system operating normally, the tap changing control is needed to restore the converter quantities, especially the angle and the power factor, to their normal range [10].

Hence, when the adjustment on $A$ cannot satisfy normal computation conditions, it can enlarge the controllable range of the DC system by fixing $A$ to its value in the previous iteration or a preset value while relaxing $T$ as a state variable. That is, the approach presented in this paper represents the characteristics of the DC control system.

\section{B. Robustness Analysis}

According to (3)-(5), if the AC bus voltage magnitude is known, the partial derivatives of (2) with respect to state variables are constant.

Equation (5) shows that, if there is no CP control mode, the partial derivatives of (5) with respect to state variables is a unit vector. That is, only one element is 1 and the others are 0 . For the $\mathrm{CP}$ control mode, the partial derivatives with respect to $V_{d i}$ and $I_{d i}$ are the corresponding $I_{d i}$ and $V_{d i}$, respectively. The partial derivatives with respect to other state variables are 0 .

Take (6) as an example. The Jacobian matrix of a two-terminal DC system is shown in (27) at the bottom of the page.

In the 3 rd and 6 th lines, the positions of the nonzero elements are different for different control modes. It is clear that for all of the four operation modes, $\boldsymbol{J}$ is always invertible.
TABLE I

THREE-TERMINAL DC SYSTEM PARAMETERS

\begin{tabular}{ccccccc}
\hline \hline Converter No. & AC bus No. & $T$ (p.u.) & Type & $X_{c}$ (p.u.) & DC line & $R_{d}$ (p.u.) \\
\hline 1 & 2 & 1.00 & $\mathrm{R}$ & 0.075 & $1-2$ & 0.07162 \\
2 & 4 & 0.99 & $\mathrm{I}$ & 0.125 & $1-3$ & 0.07162 \\
3 & 5 & 0.98 & $\mathrm{R}$ & 0.125 & $2-3$ & 0.07162 \\
\hline \hline
\end{tabular}

$T=$ Tap ratio of converter transformer, $\mathrm{R}=$ Rectifier, I=Inverter, p.u.=per unit, $X_{c}=$ Converter equivalent reactance, $R_{d}=$ the conductance of the DC line.

It is clear from the analysis above that most elements in Jacobian matrix of DC system are constant during calculation. Only for CP control mode would the corresponding elements of Jacobian matrix be modified and the inversion of $\boldsymbol{J}$ should be recalculated at each step.

Generally, $\boldsymbol{J}$ is neither symmetrical nor diagonally dominant (the diagonal element might even be zero). At present, the majority of the commercial operational DC systems are two-terminal and the maximum terminal number for DC system is only three. Therefore, for each DC system unit, the rank of $\boldsymbol{J}$ is not large (for $n$-terminal DC system, the rank is $3 n$ ). It is easy to get the inverse of $\boldsymbol{J}$ because of its small dimension. Additionally, if there is no CP control in DC system, $\boldsymbol{J}$ is a constant matrix and we only need to calculate its inverse once during the whole calculation.

The improved approach presented in this paper works well for $\mathrm{N}-1$ scanning in AC-DC power flow of the actual physical model of CSG. In most cases, the state variables do not need to switch from $A$ to $T$ under the various operations such as line outage, power or voltage change, control mode or parameter change, monopolar or bipolar blocking in DC system, etc. If it is necessary to switch the state variables, the tap ratio is usually adjusted once only during the calculation of AC-DC power flow.

\section{Simulation Results}

\section{A. The 14-Bus System Including Three-Terminal DC System}

The simulation system is modified from the IEEE 14-bus system. Three AC lines connected to buses 2, 4, and 5 are replaced by a three-terminal DC system. The control mode of the DC system is shown in Table I. The initial value of direct voltage, direct current, and $\mathrm{AC}$ bus voltage magnitudes are 1.0 per unit. The convergence precisions of iteration for AC and DC systems, $\varepsilon$, are both set as $10^{-5}$.

The number of iterations until convergence for the AC-DC hybrid power system is less than 10 and that of the DC system is less than 3 . If the tap position of the converter transformer is unsuitable, the algorithm relaxes $T$ for only once during the calculation of AC-DC power flow.

$$
\boldsymbol{J}=\left[\begin{array}{cccccc}
1 & \frac{3}{\pi} X_{c i} \zeta_{i} & -\frac{3 \sqrt{2}}{\pi} B_{i} T_{i} U_{a i} & 0 & 0 & 0 \\
-1 / R_{d i j} & 1 & 0 & 1 / R_{d i j} & 0 & 0 \\
I_{d i} & V_{d i} & 0 & 0 & 0 & 0 \\
0 & 0 & 0 & 1 & \frac{3}{\pi} X_{c j} \zeta_{j} & -\frac{3 \sqrt{2}}{\pi} B_{j} T_{j} U_{a j} \\
1 / R_{d i j} & 0 & 0 & -1 / R_{d i j} & 1 & 0 \\
0 & 0 & 0 & 0 & 0 & 1
\end{array}\right]
$$


TABLE II

CSG IN MARCH 2008

\begin{tabular}{cc}
\hline \hline Power System resources & Number \\
\hline substations & 643 \\
breakers & 7221 \\
disconnectors & 17632 \\
ground disconnectors & 9571 \\
AC lines & 2426 \\
transformers & 738 \\
compensators & 1104 \\
synchronous machines & 418 \\
energy consumers & 1217 \\
converters & 16 \\
DC lines & 8 \\
\hline \hline
\end{tabular}

TABLE III

DC LINES IN CSG IN MARCH 2008

\begin{tabular}{cccc}
\hline \hline Index & DC system & Ternimal 1 & Terminal 2 \\
\hline 1 & Gao-Zhao Pole I & Gaopo & Zhaoqing \\
\hline 2 & Gao-Zhao Pole II & Gaopo & Zhaoqing \\
3 & Xing-An Pole I & Xingren & Baoan \\
4 & Xing-An Pole II & Xingren & Baoan \\
5 & Tiang-Guang Pole I & Mawo & Guangzhou \\
6 & Tiang-Guang Pole II & Mawo & Guangzhou \\
7 & San-Guang Pole I & Jingzhou & Echeng \\
8 & San-Guang Pole II & Jingzhou & Echeng \\
\hline \hline
\end{tabular}

It shall be noted that the calculation might fail for a three-terminal DC system with two converters operating under CA control mode and one converter under $\mathrm{CP}$ control mode. If the initial values of $\mathrm{AC}$ bus voltages are good, the calculation may converge successfully. Otherwise, it will diverge even if the N-R method is adopted to calculate AC system with Jacobian matrix revised instead of the PQ decoupled method. Furthermore, if the quasi-Newton method is used to conquer the problems caused by ill-conditioned feature of the Jacobian matrix in this situation, the DC part may converge. The AC part, however, would still diverge sometimes. The phenomenon originates from the physical characteristic of the system instead of the mathematical algorithm. When a three-terminal DC system with dc converter stations connected in series on the DC side operates with the control mode mentioned above, the operating point is essentially unstable. Therefore, the iteration is hard to converge.

\section{B. China Southern Power Grid}

The network of CSG in March 2008 has five control areas, including Guangdong Power Grid, Guangxi Power Grid, Guizhou Power Grid, Yunnan Power Grid, and Hainan Power Grid. The details of the system are shown in Tables II and III.

For a two-terminal DC system, the "ill-conditioned" situation does not appear under any control combination, and it converges rapidly. To illustrate the stability of the proposed approach, seven cases are selected from Table IV. Other cases, including Case 8, have shown similar performance.

The initial control mode of DC systems is CP in rectifier and $\mathrm{CA}$ in inverter. The power flow of the system is calculated according to state estimation results.
TABLE IV

OPERATIONS ON THE SIMULATION SYSTEM

\begin{tabular}{cc}
\hline \hline Case & Operation \\
\hline 1 & Gao-Zhao monopolar Blocking \\
2 & Gao-Zhao bipolar Blocking \\
3 & The extinction angle of Baoan Pole II increase $8^{\circ}$ \\
4 & The power of Tian-Guang Pole I decrease $1000 \mathrm{MW}$ \\
5 & Reduction of the AC voltage magnitude of Xingren by $30 \%$ \\
6 & Outage of the ECheng Converter \\
7 & Outage of the DC line of Xing-Bao Pole I \\
8 & Various control combinations \\
\hline \hline
\end{tabular}

TABLE V

POWER FLOW RESULTS

\begin{tabular}{ccc}
\hline \hline Operation & $\begin{array}{c}\text { The number of } \\
\text { iterations for } \\
\text { AC system } \\
(P, Q)\end{array}$ & $\begin{array}{c}\text { The Average number } \\
\text { of DC system iterations } \\
\text { until convergence }\end{array}$ \\
\hline According to SE result & 4,5 & 1.2 \\
1 & 5,5 & 1.8 \\
2 & 9,9 & 1.5 \\
3 & 6,6 & 1.3 \\
4 & 8,8 & 1.5 \\
5 & 6,6 & 1.8 \\
6 & 8,9 & 1.4 \\
7 & 9,9 & 1.6 \\
\hline \hline
\end{tabular}

$\mathrm{SE}=$ State Estimation, $\mathrm{P}, \mathrm{Q}=$ in this column, the first number is the number of iterations of $\mathrm{P} \theta$ part, and the second is of $\mathrm{QV}$.

TABLE VI

COMPARISON With THE TRADITIONAL METHOD

\begin{tabular}{ccc}
\hline \hline Operation & $\begin{array}{c}\text { Proposed approach } \\
\text { in this paper }\end{array}$ & $\begin{array}{c}\text { Traditional } \\
\text { sequential method }\end{array}$ \\
\hline According to SE result & Converge & converge \\
1 & Converge & converge \\
2 & Converge & Diverge \\
3 & Converge & Converge \\
4 & Converge & Diverge \\
5 & Converge & Diverge \\
6 & Converge & Diverge \\
7 & Converge & Diverge \\
\hline \hline
\end{tabular}

Many experiments have been done to test this proposed approach, including power reduction, voltage reduction, line outage, monopolar blocking, bipolar blocking, control parameter adjustment, control mode shift, and other combined operations. The simulations almost covered all the adjustments and operations of DC systems.

Power flow is calculated directly by using the improved approach proposed in this paper. The results are satisfactory as shown in Table $\mathrm{V}$.

The number of alternative iterations is less than ten, and the average number of DC iterations is less than two. The convergence precision is $10^{-5}$. The results of $\mathrm{N}-1$ contingency scan also show that the algorithm is robust.

Table VI illustrates the comparison with the traditional sequential method for calculation of AC-DC power flow. It shows 
that the algorithm proposed in this paper works well for the different control modes that can lead to a stable operating point with a rapid speed and accurate results.

\section{CONCLUSION}

To enhance the robustness of AC-DC power flow calculation, an improved sequential approach for multi-infeed DC systems is proposed in this paper. It avoids unfeasible solutions, interruptions, and oscillations during AC-DC power flow calculations. Theoretical analyses proved that the new approach indeed has such advantages. Besides, the new approach has the following favorable properties:

1) Regards each individual DC system as a calculation unit. This approach makes the calculation processes of all DC systems independent to each other. Therefore, the scale of calculation equations is significantly reduced.

2) Uses the Newton-Raphson method to calculate the DC systems. The Jacobian matrix maintains nonsingular under various control modes. It is also convenient for the exchange of state variables.

3) Ensures $\cos \theta<1$ and flexibly adjusting of the tap position of converter transformer. It can avoid taking square root of negative numbers and numerical oscillations during AC-DC power flow calculations.

4) Adopts an alternative iterative method to make full use of $\mathrm{AC}$ power flow program widely accepted, so it is easy to program.

Numerical simulations and practical applications show that the approach presented in this paper has advantages in terms of convergence and speed. This new approach can also accommodate the continual development of CSG. For example, a new DC line, Gui-Guang Stage II, was incorporated into CSG successfully in December 2007 without changing the power flow calculation in the existing systems.

Since its implementation in the EMS of CSG in September 2007, the program module of AC-DC power flow calculation based on the improved approach has been called every $5 \mathrm{~min}$. The results of this module are essential to many other functions of EMS [13], such as steady security analysis, network sensitivity analysis, transmission limit, optimal power flow, automatic voltage control, automatic generation control, etc. As a result, the improvement on power flow calculation proposed in this paper significantly benefits the whole EMS system.

\section{ACKNOWLEDGMENT}

The authors would like to thank Dr. K. Liu and Dr. Y. Lin of the Department of Electrical Engineering, the University of Hong Kong, for their helpful discussion.

\section{REFERENCES}

[1] J. Yu, W. Yan, W. Y. Li, C. Y. Chung, and K. P. Wong, "An unfixed piecewise-optimal reactive power-flow model and its algorithm for AC-DC systems," IEEE Trans. Power Syst., vol. 23, no. 1, pp. 170-176, Feb. 2008.

[2] Y. Y. Sun, G. B. Zhang, W. Xu, and J. G. Mayordomo, "A harmonically coupled admittance matrix model for AC/DC converters," IEEE Trans. Power Syst., vol. 22, no. 4, pp. 1574-1582, Nov. 2007.
[3] J. R. D. Silva and C. P. Arnold, "A simple improvement to sequential AC/DC power flow algorithms," Elect. Power Energy Syst., vol. 12, no. 3, pp. 219-221, Jul. 1990.

[4] G. D. Prasad and P. S. Rao, "A heuristic method for the real-time load flow solution of integrated multi-terminal AC-DC power systems," Elect. Power Syst. Res., vol. 28, pp. 139-147, 1993.

[5] K. R. Padiyar and V. Kalyanaraman, "Power flow analysis in MTDC-AC systems-A new approach," Elect. Mach. Power Syst., vol. 22, pp. 271-288, 1994.

[6] T. Smed, A. G. Andersson, G. B. Sheble, and L. L. Grigsby, "A new approach to AC/DC power flow," IEEE Trans. Power Syst., vol. 6, no. 3, pp. 1238-1244, Aug. 1991.

[7] Y. K. Fan, D. Niebur, C. O. Nwankpa, H. Kwatny, and R. Fischl, "Multiple power flow solutions of small integrated AC/DC power systems," in Proc. IEEE 2000 Int. Symp. Circuits and Systems, Geneva, Switzerland, 2000, pp. 224-227.

[8] Q. F. Ding and B. M. Zhang, "A new approach to AC/MTDC power flow," in Proc. 4th Int. Conf. Advances in Power System Control, Operation and Management (APSCOM-97), Hong Kong, 1997, pp. 689-694.

[9] J. Arrillage and N. A. Watson, Computer Modelling of Electrical Power Systems, 2nd ed. Chichester, U.K.: Wiley, 2001, pp. 135-146.

[10] K. Parbha, Power System Stability and Control. New York: McGrawHill, 1993, pp. 545-551.

[11] B. M. Zhang, S. S. Chen, and Z. Yan, Advanced Power System Network Analysis, 2nd ed. Beijing, China: Tsinghua Univ. Press, 2007, pp. 194-199.

[12] C. A. Canizares and F. L. Alvarado, "Point of collapse and continuation methods for large AC/DC systems," IEEE Trans. Power Syst., vol. 8, no. 1, pp. 1-8, Feb. 1993.

[13] B. M. Zhang, H. B. Sun, and W. C. Wu, "A new generation of EMS implemented in Chinese electric power control centers," in Proc. 2008 IEEE Power and Energy Soc. General Meeting_Conversion and Delivery of Electrical Energy in the 21st Century, Pittsburgh, PA, Jul. 20-24, 2008, pp. 1-3.

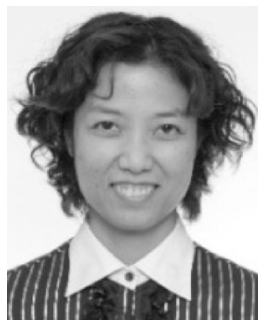

Chongru Liu (M'10) received the B.S., M.S., and $\mathrm{Ph} . \mathrm{D}$. degrees in electrical engineering from Tsinghua University, Beijing, China, in 2000, 2003, and 2006, respectively.

She is now an Associate Professor in the School of Electrical and Electronic Engineering, North China Electric Power University, Beijing. She has been working on several funded research projects, such as the dynamic simulation model of DC system, the research on power optimization and coordinated control of AC-DC hybrid power transmission corridor, the research on AC-DC system state estimation based on PMU data, the characteristics of DC system and the control strategy for multi-infeed DC systems, etc. Her current interests include the analysis, operation, and control of AC-DC systems.

Dr. Liu is a member of the National Power System Management and Information Exchange Standardization Committee of China.

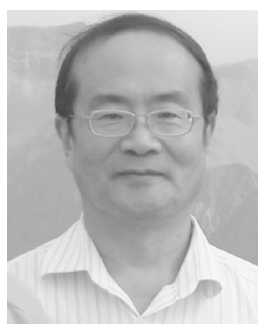

Boming Zhang (SM'95-F'10) received the Ph.D. degree in electrical engineering from Tsinghua University, Beijing, China, in 1985.

Since 1985, he has been with the Electrical Engineering Department, Tsinghua University, for teaching and research and promoted to a Professor in 1993. His interest is in power system analysis and control, especially in the EMS advanced applications in the Electric Power Control Center (EPCC). He has published more than 300 academic papers and implemented more than 60 EMS/DTS systems in China. He is now a steering member of CIGRE China State Committee and of the International Workshop of EPCC. 


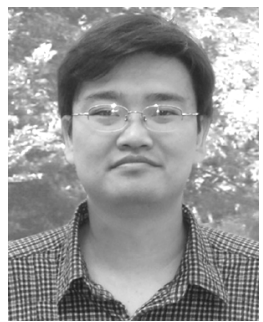

Yunhe Hou (M'06) received the B.E. and Ph.D. degrees from Huazhong University of Science and Technology, Wuhan, China, in 1999 and 2005, respectively.

He worked as a postdoctoral research fellow at Tsinghua University, Beijing, China, from 2005 to 2007. He was a visiting scholar at Iowa State University, Ames, and a research scientist at University College Dublin, Ireland, from 2008 to 2009. He is currently with the University of Hong Kong as a Research Assistant Professor.

Felix F. Wu (M'74-F'89) is the Philip Wong Wilson Wong Professor in Electrical Engineering at the University of Hong Kong, where he served as Pro Vice Chancellor (Vice President) from 1997 to 2001. He is also a Professor Emeritus at the University of California, Berkeley, where he has been on the faculty since 1974.

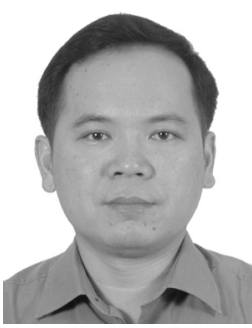

Yingshang Liu received the M.Ens.Sc. degree from South China University of Technology, Guangzhou, Guangdong, China, in 1987 and the Ph.D. degree from Tsinghua University, Beijing, China, in 2006, both in electrical engineering.

$\mathrm{He}$ has 17 years of working experience in the Power System Control Center. Since 2004, he has worked for the Department of Production and Technology of China Southern Power Grid Company, Guangzhou, China. He is now a professor-level senior engineer. His interests include power system operation, emergency control and asset management, etc.

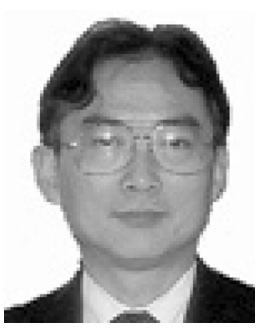

